\title{
The inverse light-bulb
}

\author{
IMAGE \\ UNAVAILABLE FOR \\ COPYRIGHT \\ REASONS
}

Progress from crystallographic data to resolved protein structure is now often only a matter of months but, for some structures, the magnitude of the problem means that the process is spread over a number of years. Such is the case with the structure of the photosynthetic reaction centre from the purple bacterium Rhodopseudomonas viridis pictured here (Deisenhofer, J., Epp, O., Sinning, I. \& Michel, H., J.molec.Biol. In the press). This protein was the first integral membrane complex for which crystals suitable for crystallographic analyses were obtained over thirteen years ago. Since then a single group has refined the structure to its present detailed model at $2.3 \AA$ resolution and an $R$-factor of $19 \%$.

The photosynthetic reaction centre performs the primary steps in photosynthesis. Light energy, collected by separate light-harvesting antenna complexes, is converted into electrochemical energy in the form of a proton gradient across the membrane in which the protein complex is embedded. The complex is made up from four proteins (shown in different colours), two of which are integral membrane proteins containing a number of membrane spanning helices. There are 14 cofactors (carbon atoms coloured white), including four haems, two quinones and a carotenoid, and also a number of water molecules which may have functional roles being positioned to take part in electron transfer between the various cofactors.

Fixed water molecules were also a feature of the related, although less complex, photosynthetic reaction centre from Rhodobacter sphaeroides (Ermler, U., Fritzsch, G., Buchanan, S.K. \& Michel, H. Structure 2 925-936). In that case a continuous string of 14 water molecules connects the secondary quinone cofactor to the surface of the protein and may act as a proton wire.

The structure of the photosynthetic reaction centres from purple bacteria have provided a framework for the interpretation of data from other sources and the understanding of the mechanism of photosynthesis both in bacteria and in other organisms. The increasing detail in these structures can only make this understanding more complete. Unfortunately the determination of such large protein complexes is a long process requiring unhampered vision and long-term support. 